\title{
Selection of reference genes for gene expression studies in human bladder cancer using SYBR-Green quantitative polymerase chain reaction
}

\author{
CHUANXIA ZHANG ${ }^{1,2}$, YONG QIANG WANG ${ }^{3}$, GUANGYI JIN ${ }^{4}$, SONG WU ${ }^{5}$, JUN CUI ${ }^{1,6}$ and RONG-FU WANG ${ }^{7-9}$ \\ ${ }^{1}$ Key Laboratory of Gene Engineering of The Ministry of Education, State Key Laboratory of Biocontrol, School of Life \\ Sciences, Sun Yat-sen University, Guangzhou, Guangdong 510275; ${ }^{2}$ Zhongshan School of Medicine; ${ }^{3}$ Department \\ of Urology, Sun Yat-sen University Cancer Center, Collaborative Innovation Center for Cancer Medicine, State Key \\ Laboratory of Oncology in South China, Guangzhou, Guangdong 510060; ${ }^{4}$ Shenzhen Key Laboratory of Translational \\ Medicine of Tumor, Cancer Research Center, School of Medicine, Shenzhen University, Shenzhen, Guangdong 518060; \\ ${ }^{5}$ Department of Urological Surgery, Shenzhen Second People's Hospital, The First Affiliated Hospital of Shenzhen \\ University; ${ }^{6}$ Collaborative Innovation Center of Cancer Medicine, Sun Yat-sen University, Guangzhou, Guangdong \\ 510275, P.R. China; ${ }^{7}$ Center for Inflammation and Epigenetics, Houston Methodist Research Institute, Houston, TX 77030; \\ ${ }^{8}$ Department of Microbiology and Immunology, Weill Cornell Medical College, Cornell University, New York, NY 10065; \\ ${ }^{9}$ Institute of Biosciences and Technology, College of Medicine, Texas A \& M University, Houston, TX 77030, USA
}

Received February 28, 2016; Accepted July 20, 2017

DOI: $10.3892 / \mathrm{ol} .2017 .7002$

\begin{abstract}
Reverse transcription-quantitative polymerase chain reaction (RT-qPCR) is a rapid, reliable and widely used method of studying gene expression profiles that requires appropriate normalization for accurate and reliable results. Reference genes are usually used to normalize mRNA levels; however, the expression levels of these reference genes may vary between cell types, developmental stages, species and experimental conditions. Therefore, a normalization strategy is an important precondition for reliable conclusions, with endogenous controls requiring determination for every experimental system. In the present study, 18 reference genes used in various prior studies were analyzed to determine their applicability in bladder cancer. A total of 35 matched malignant and non-malignant bladder cancer (specifically transitional cell carcinoma) tissue specimens were examined. RNA and cDNA quality was stringently controlled. Candidate reference genes were assessed using SYBR-Green RT-qPCR. mRNA abundance was compared and
\end{abstract}

Correspondence to: Professor Jun Cui, Key Laboratory of Gene Engineering of The Ministry of Education, State Key Laboratory of Biocontrol, School of Life Sciences, Sun Yat-sen University, 135 Xingang Xi Road, Guangzhou, Guangdong 510275, P.R. China E-mail: cuij5@mail.sysu.edu.cn

Professor Rong-Fu Wang, Center for Inflammation and Epigenetics, Houston Methodist Research Institute, 6670 Betner Avenue, Houston, TX 77096, USA

E-mail: rwang3@houstonmethodist.org

Key words: SYBR Green quantitative polymerase chain reaction, reference gene, bladder cancer, quantification cycle value, biomarker reference genes with distinct ranges of expression to possible target genes were excluded. Genes that were differentially expressed in matched non-cancerous and cancerous samples were also excluded, using quantification cycle analysis. Subsequently, the stability of the selected reference genes was analyzed using three different methods: geNorm, NormFinder and BestKeeper. The rarely used ribosomal protein S23 (RPS23) was the most stable single reference gene, with RPS23, tumor protein, translationally controlled 1 and RPS13 comprising the optimal reference gene set for all the bladder samples. These stable reference genes should be employed in normalization and quantification of transcript levels in future expression studies of bladder cancer-associated genes.

\section{Introduction}

Bladder cancer, a disease that is reported more frequently in men than in women, is the most common urological malignancy (1). It has been estimated that there were 74,000 novel cases and 16,000 incidences of mortality in the USA alone in 2015 (2). In total, $90 \%$ of these patients were diagnosed with transitional cell carcinoma (TCC), whereas adenocarcinoma and squamous cell carcinoma accounted for $<10 \%$ of the cases (3). TCC is one of only a limited number of types of cancer that is responsive to immunotherapy (4). Bladder cancer has a high probability of recurrence, which makes it a costly cancer to treat (5). To develop an effective and cost-effective therapy for TCC, it is necessary to identify and exploit the molecular mechanisms and factors involved (6), particularly the markers of recurrent disease.

Comparing the level of certain mRNAs in cancer tissues with the same patents' normal tissues is a practical approach to identifying new biomarkers implicated in the TCC process. 
In order to do this, fluorescence-based reverse transcription-quantitative polymerase chain reaction (RT-qPCR), which is one of the most common and powerful quantification methods, was employed in the present study to evaluate mRNA expression in specimens affected by bladder cancer. The result obtained by RT-qPCR not only informs on the cancer-driven biological variation of gene expression, but also reflects the confounding factors as well. According to The Minimum Information for Publication of Quantitative Real-Time PCR Experiments (MIQE) guidelines (7), these factors are associated with the entire procedure of qPCR, from experimental design to data analysis. Therefore, the choice of reference genes is one of the most influential factors in RT-qPCR, because these genes can be applied to a normalization strategy to monitor the variation in amplification efficiencies as well as the differences between the samples, particularly for cancer tissues (8).

Reference genes, such as the housekeeping genes GAPDH and $\beta$-actin, are usually constitutively expressed at high levels in different cell types or tissues, but their mRNA levels may be affected by the cell type or tissue used, as well as the experimental conditions $(9,10)$. Thus, it is of critical importance to evaluate the reference genes under similar experimental conditions in the same tissue type prior to their use normalizing RNA levels for target genes. Several freely available mathematical software programs, including geNorm (11), NormFinder (12) and BestKeeper (13), have been developed to address this issue.

Despite the fact that the selection of a reference gene is important, the majority of published gene expression reports do not state the rationale for the selection made (14). As a result, numerous studies of gene expression often fail to produce comparable and reliable results. RT-qPCR with SYBR-Green is widely used in a number of studies investigating cancer, but few have reported the expression stability of reference genes in bladder cancer $(12,15)$. One study reportedly employed SYBR-Green chemistry (12), but it was performed without any matched non-cancerous samples and used different mathematical algorithms from those in the present study. Therefore, the present study sought to identity the most suitable reference genes for mRNA profiling in bladder cancer, specifically TCC. To this end, 18 carefully selected reference genes were quantified using SYBR-Green qPCR, then the expression stability of the candidate genes was evaluated using quantification cycle (Cq) analysis, geNorm, NormFinder and BestKeeper.

\section{Materials and methods}

Human TCC sample collection. The inclusion criteria were as follows: Patients newly diagnosed with TCC for whom tumors were removed by transurethral resection and patients with adequately matched non-cancerous TCC samples. Exclusion criteria included non-TCC histology, salvage cystectomy, upper tract TCC and incomplete medical records. Written informed consent was obtained from all patients and the present study was approved by the Sun Yat-Sen University Cancer Center (Guangzhou, China) and the institutional review board of Shenzhen Second People's Hospital (Shenzhen, China). A total of 35 fresh tumor samples with matched normal controls (morphologically adjacent normal bladder tissues)
Table I. Characteristics of transitional cell carcinoma samples.

Characteristic

Patients, $\mathrm{n}$

Total number of patients 35

Age

Mean (range)

$61(41-81)$

Sex

Male

30

Female

5

Histological grade

Low

High

20

Unknown

Tumor stage ${ }^{\mathrm{a}}$

pTa

2

pT1

12

pT2

7

pT3

6

pT4

5

Unknown

Lymph node status

Negative

29

Positive

3

Unknown

Metastasis

Negative

31

Positive

1

Unknown

${ }^{\text {aTumors }}$ were staged according to tumor-node-metastasis system recommended by the American Joint Committee on Cancer (25). Each sample had matched cancerous and non-cancerous specimens.

were obtained from individuals newly diagnosed with TCC from June 2013 to October 2014 (Table I). Patients were aged between 41 and 81 years, with a mean age of 61 years. The samples were divided into two groups: One group was used for conventional pathology examination analysis in the pathology department of the hospital, whereas the second was immediately immersed in RNAlater (Qiagen $\mathrm{GmbH}$, Hilden, Germany) and stored at $-80^{\circ} \mathrm{C}$ or snap-frozen in liquid nitrogen until RNA extraction.

Total RNA isolation and cDNA synthesis. The preserved TCC samples (18-70 mg) were cut into the smallest possible pieces and homogenized in $200 \mu \mathrm{l}$ TRIzol reagent (Invitrogen; Thermo Fisher Scientific, Inc., Waltham, MA, USA) under sterile conditions. Total RNA was then extracted according to the manufacturer's protocol. The quantification and quality control of total RNA were performed in triplicate with a NanoDrop2000 spectrophotometer (Thermo Fisher Scientific, Inc.). According to the MIQE guidelines, the following conditions must be met for cDNA synthesis: An RNA concentration $>50 \mathrm{ng} / \mu \mathrm{l}$ and absorbance ratios of 260/280 $\mathrm{nm}$ of between 1.8 and 2.1. The integrity and genomic DNA contaminant of isolated RNA was 


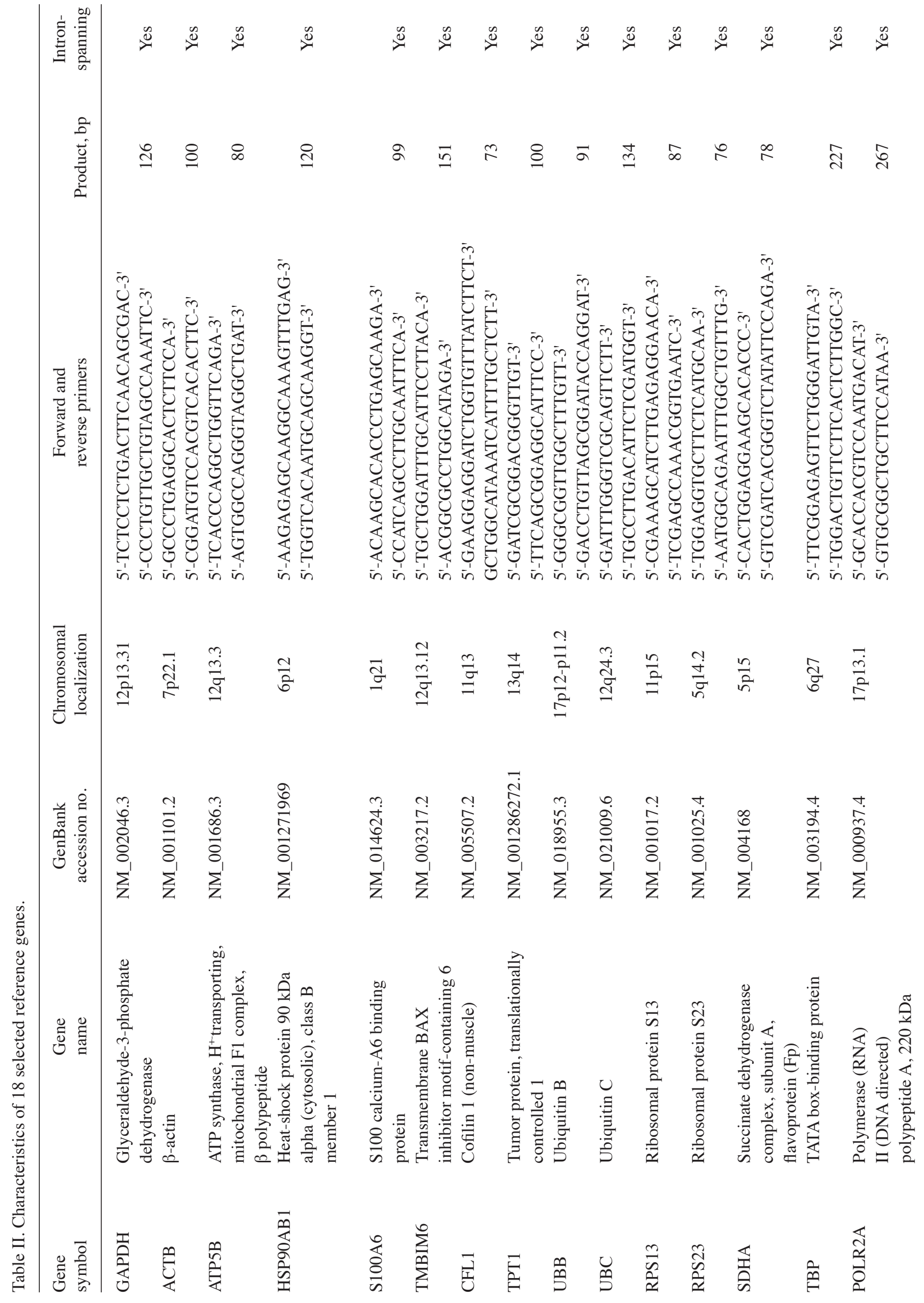


confirmed by $1.2 \%$ SYBR-Green-safe agarose electrophoresis (Invitrogen; Thermo Fisher Scientific, Inc.).

In a $20 \mu \mathrm{l}$ PCR, $1 \mu \mathrm{g}$ of Total RNA was reverse-transcribed into first-strand cDNA using PrimeScript RT Master Mix (Takara Bio, Inc., Otsu, Japan). The reaction mixture was incubated at $37^{\circ} \mathrm{C}$ for $15 \mathrm{~min}$ for RT, then at $85^{\circ} \mathrm{C}$ for $5 \mathrm{sec}$ to inactivate reverse transcriptase. The RT products were subsequently stored at $-20^{\circ} \mathrm{C}$ until further use. The PrimeScript RT Master mix included reaction buffer $\left(\mathrm{Mg}^{2+}\right)$, PrimeScript reverse transcriptase, dNTP mixture, random hexamers, oligo dT primer and an RNase inhibitor, used to ensure the uniformity of the reverse transcription reaction between samples. cDNAs of all samples were diluted 1:10 for qRT-PCR.

Selection of the candidate reference genes and validation of the primers. A total of 18 candidate reference genes were selected in the present study. Their identity and characteristics are summarized in Table II, including the gene name, GenBank accession number and the sequence of primer pairs.

The primers were synthesized by Sangon Biotech Co., Ltd (Shanghai,China). They were selected on the basis of published reports on reference gene expression profiles and previous databases (11-16). Next, the primer sequences were cross-checked using the University of California Santa Cruz web-based tool in silico PCR (genome.ucsc.edu/cgi-bin/hgPcr) (17) against genomic and gene targets. qPCR was performed on cDNA of randomly selected tumor tissues to check the specificity of all primers. The melting curve and the visualized PCR products on $2 \%$ SYBR-Green-safe agarose gel were then evaluated (Fig. 1).

$R T$ - $q P C R$. RT-qPCR for 18 candidate reference genes was performed in 96-well plates with the LightCycler 480 Real-Time PCR System (Roche Applied Science, Pleasanton, CA, USA) and qTOWER2.0 (Analytik Jena AG, Jena, Germany). A LightCycler 480 SYBR Master (Roche Applied Science) was used to detect double-stranded DNA synthesis. In a $10 \mu$ l total volume, the PCRs contained $5 \mu 1$ Premix Ex Taq (Roche Applied Science), $1 \mu 1$ 10-fold diluted RT product (50 ng total RNA), $1 \mu 1$ forward and $1 \mu 1$ reverse PCR primers (300 nM; Sangon Biotech Co., Ltd) and $2 \mu$ nuclease-free water (Roche Applied Science). The reaction mixtures were processed with an initial holding period at $95^{\circ} \mathrm{C}$ for $10 \mathrm{~min}$, followed by a three-step PCR program for 45 cycles that consisted of $95^{\circ} \mathrm{C}$ for $10 \mathrm{sec}, 60^{\circ} \mathrm{C}$ for $10 \mathrm{sec}$ and $72^{\circ} \mathrm{C}$ for $25 \mathrm{sec}$. Immediately following PCR, a melting curve program was activated by heating the product from $65-95^{\circ} \mathrm{C}$ at $0.1^{\circ} \mathrm{C}$ intervals. Reverse transcriptase negative controls and no-template controls (NTC) were also included in each experiment to avoid the contamination of genomic DNA or primer dimers. Cancerous and corresponding non-cancerous specimens were processed in the same run to exclude between-run variations. The results of the melting curve analysis and electrophoresis of the PCR products were used to confirm the specificity of the amplification for each of the primer pairs (Fig. 1).

Cq value calculation and $P C R$ efficiency. The Cq value is equal to the number of cycles at which the value of the fluorescent signal reaches a given threshold of detection, and this value is negatively associated with the initial amount of the input 

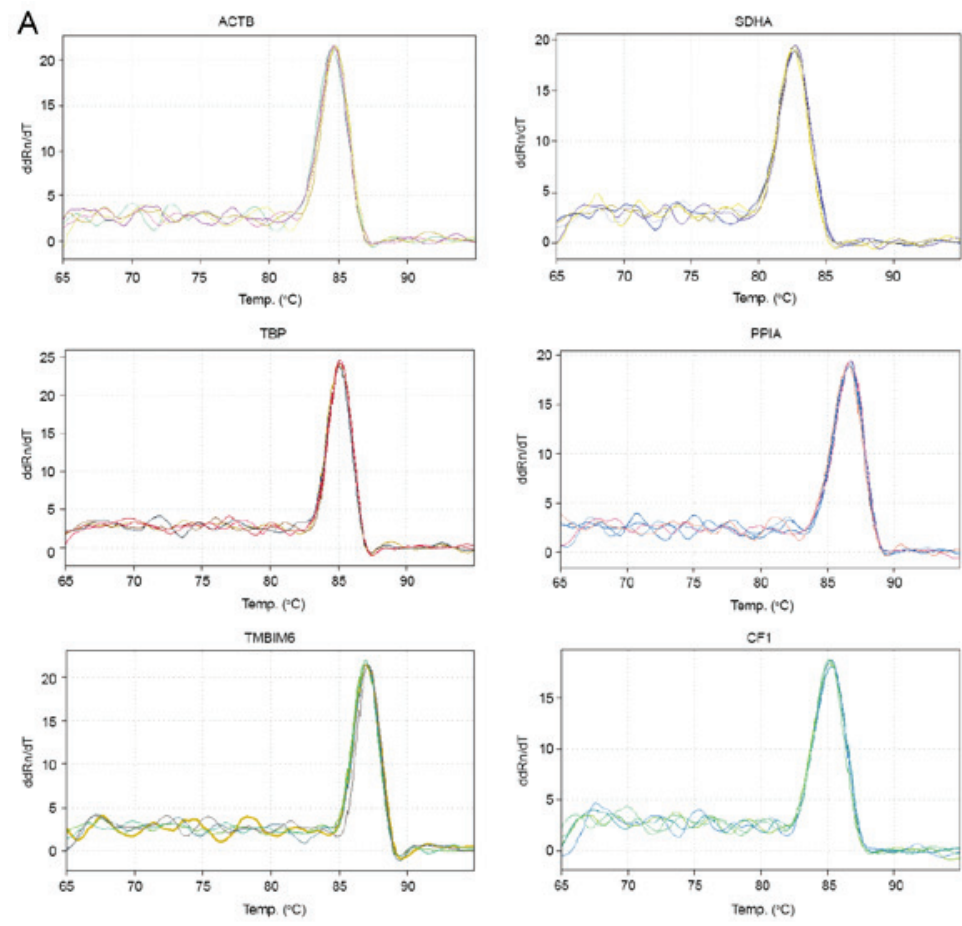

S10046
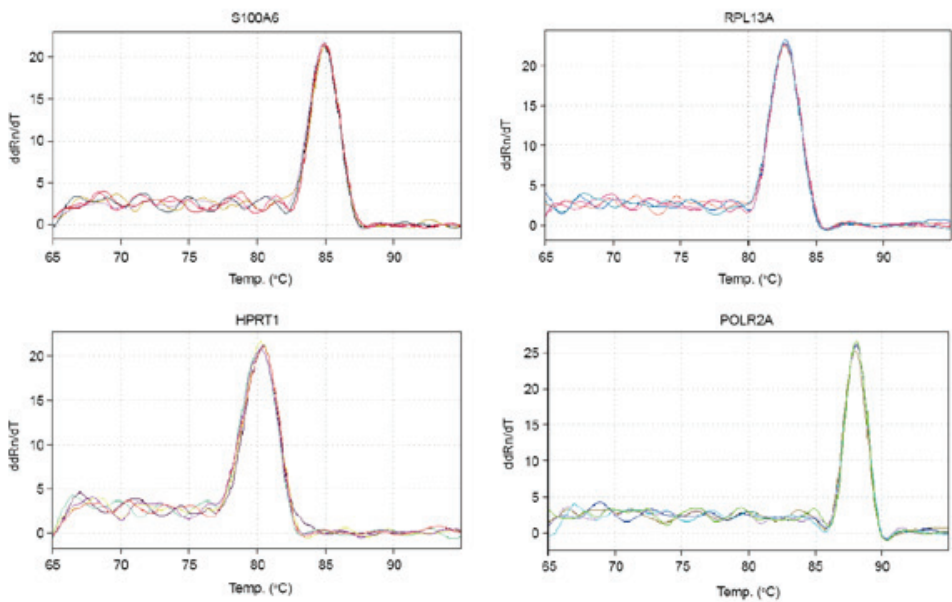

บBs
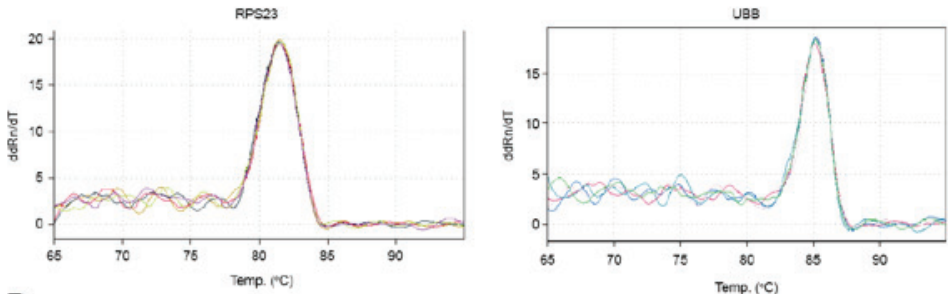
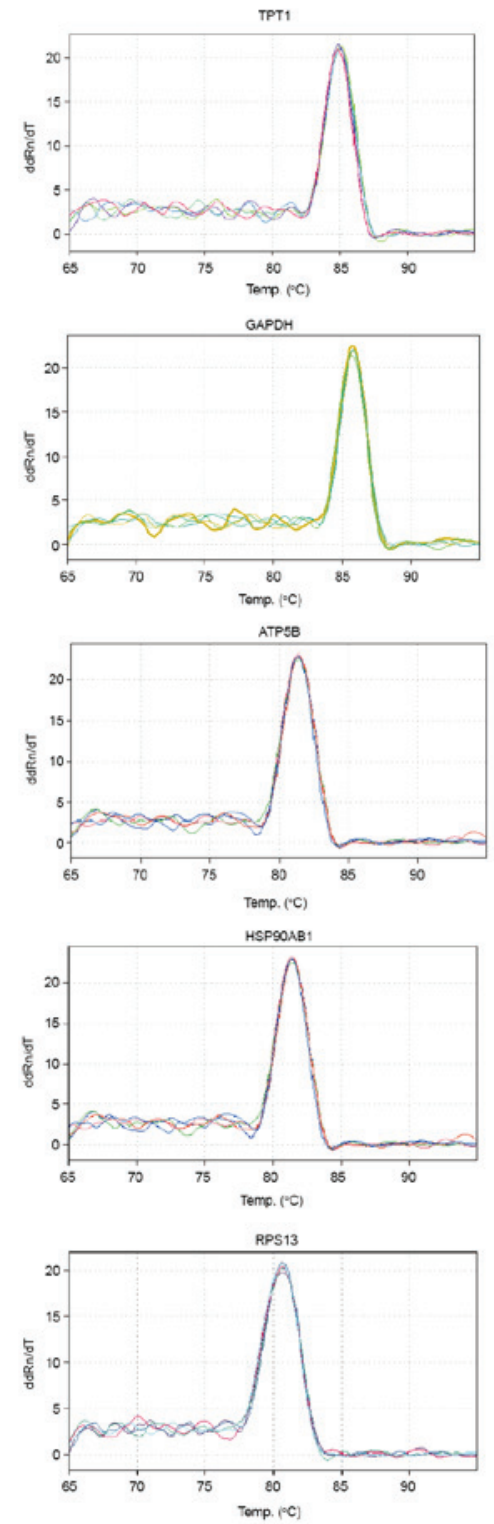

Uac

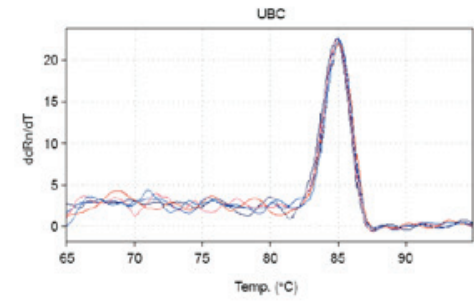

B

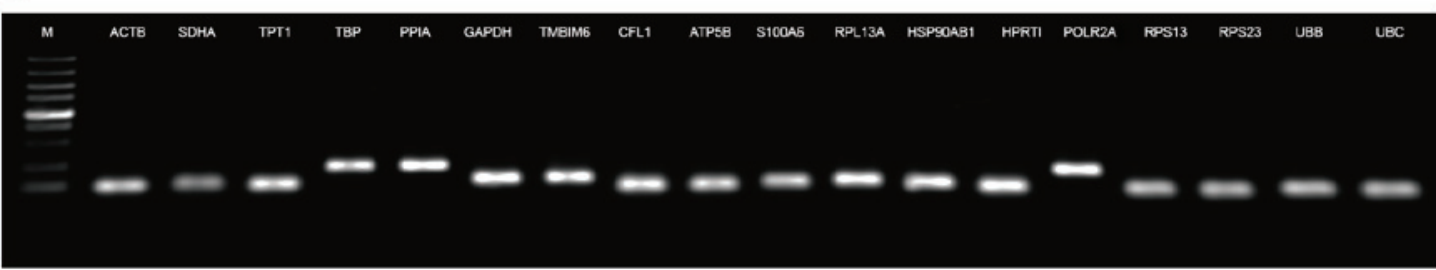

Figure 1. Primer specificity analysis for the 18 candidate reference genes. (A) Melting curves of the 18 reference genes exhibiting single peaks. (B) Agarose gel electrophoresis (1.2\%) exhibited a single and specific polymerase chain reaction product of each reference gene. M, marker lane (from bottom to top: 100 , 250, 500, 750, 1,000, 1,500, 2,000, 3,000 and 5,000 bp). See Table II for gene name definitions.

mRNA $(8,18)$. In the present study, the Cq value was adjusted to 0.1 in order to compare different qPCRs on different plates, runs and days. Values were excluded from further study when the Cq value was $>40$. The gene-specific PCR efficiency of the primers were calculated from the standard curves, which were constructed using the following method: cDNA was diluted 
1:10 from $100 \mathrm{ng}$ to $1 \mathrm{pg}$ total RNA prior to reverse transcription in triplicate; following qPCR, the $\mathrm{Cq}$ values obtained for the different concentrations of the input RNA were plotted and linear regression was carried out for each selected reference gene. The efficiency $(\mathrm{E})$ was calculated using the linear equation: $\mathrm{E}=10^{(-1 / \text { slope })}-1(8,18)$. The $2^{-\Delta \Delta \mathrm{Cq}}$ analysis was based on this equation (7).

Data and statistical analysis. The stability of the reference gene candidates was evaluated with the Microsoft Excel-based (Microsoft Corporation, Redmond, WA, USA) software programs of the currently available algorithms: geNorm (11), NormFinder (12) and BestKeeper (13). The raw qPCR data were exported into Microsoft Excel files (.xls) and the $\mathrm{Cq}$ values were converted into the corresponding format to meet the requirements of the software. $\mathrm{Cq}$ values were directly subjected to BestKeeper analysis, whereas $\Delta \mathrm{Cq}$ values were required for geNorm and NormFinder analysis. The $\Delta \mathrm{Cq}$ value equals the raw $\mathrm{Cq}$ values minus the lowest $\mathrm{Cq}$ value of qPCR for each gene. The equation $\mathrm{E}^{-\Delta \mathrm{Cq}}$ was used for each data point (19). A paired Student's t-test, used to compare numerical data between the matched non-malignant and malignant specimens, was performed using GraphPad Prism (version 5.1; GraphPad, Inc., La Jolla, CA, USA). The correlation coefficients $\left(\mathrm{R}^{2}\right)$ of each primer pair were calculated from the standard curves. The coefficient of correlation (R) demonstrated gene expression variation and was determined by calculating the coefficient of variance and standard deviation of the Cq set using BestKeeper analysis (version 1.0; http://www.gene-quantification.de/bestkeeper.html).

\section{Results}

Specificity and efficiency of the primers for the 18 candidate reference genes. Melting curve analysis and agarose gel electrophoresis gave a single expected product for each selected gene (Fig. 1). No primer dimers or non-specific PCR products were detected in the NTC, and there were no evident genomic DNA contamination, as evidenced by the negative result obtained using the reverse transcriptase negative control.

The RT-qPCR efficiency of the primers was determined using a dilution of 1:10 of the cDNA template from a randomly selected human TCC sample. RNA levels may vary in the course of reverse transcription, so a randomly selected sample was selected rather than a plasmid (20-23). All the PCR assays produced efficiency values for each gene, which ranged between 1.97 and 2.15, with a slope from -3.045 to -3.392 , intercept from 26.354 to 31.279 and $\mathrm{R}^{2}$ from 0.994 to 0.999 (Table III).

Expression levels of 18 candidate reference genes. A total of 18 candidate reference genes were selected, involved in different pathways and functions, to avoid even the slightest deviation in co-regulation (11). Two cases [ribosome protein S13 (RPS13) and RPS23, ubiquitin B (UBB) and UBC] had similar functions, but were located on different chromosomes (Table I). The $\mathrm{Cq}$ values of these genes vary considerably, ranging between 17.14 [tumor protein, translationally controlled 1 (TPT1)] and 34.9 cycles [hypoxanthine phosphoribosyltransferase 1 (HPRT1)]. Furthermore, the scope of the Cq values between 19
Table III. Quantitative polymerase chain reaction parameters providing the standard curve for each primer pair.

\begin{tabular}{lllllc}
\hline Gene & Slope & Intercept & $\mathrm{R}^{2}$ & Efficiency & $\begin{array}{c}\text { Dilution } \\
\text { range }\end{array}$ \\
\hline GAPDH & -3.245 & 27.050 & 0.996 & 2.03 & $1 \mathrm{pg}-100 \mathrm{ng}$ \\
ACTB & -3.221 & 28.070 & 0.999 & 2.04 & $1 \mathrm{pg}-100 \mathrm{ng}$ \\
ATP5B & -3.045 & 26.718 & 0.999 & 2.13 & $1 \mathrm{pg}-100 \mathrm{ng}$ \\
HSP90AB1 & -3.130 & 26.354 & 0.998 & 2.10 & $1 \mathrm{pg}-100 \mathrm{ng}$ \\
S100A6 & -3.252 & 28.418 & 0.999 & 2.03 & $1 \mathrm{pg}-100 \mathrm{ng}$ \\
TMBIM6 & -3.246 & 28.059 & 0.999 & 2.03 & $1 \mathrm{pg}-100 \mathrm{ng}$ \\
CFL1 & -3.151 & 27.134 & 0.999 & 2.08 & $1 \mathrm{pg}-100 \mathrm{ng}$ \\
TPT1 & -3.264 & 27.620 & 0.999 & 2.02 & $1 \mathrm{pg}-100 \mathrm{ng}$ \\
UBB & -3.359 & 27.549 & 0.999 & 1.98 & $10 \mathrm{pg}-10 \mathrm{ng}$ \\
UBC & -3.278 & 27.316 & 0.999 & 2.02 & $1 \mathrm{pg}-100 \mathrm{ng}$ \\
RPS13 & -3.288 & 28.422 & 0.999 & 2.01 & $1 \mathrm{pg}-100 \mathrm{ng}$ \\
RPS23 & -3.199 & 27.754 & 0.999 & 2.06 & $1 \mathrm{pg}-100 \mathrm{ng}$ \\
SDHA & -3.333 & 30.257 & 0.999 & 2.01 & $10 \mathrm{pg}-10 \mathrm{ng}$ \\
TBP & -3.392 & 31.279 & 0.994 & 1.97 & $10 \mathrm{pg}-10 \mathrm{ng}$ \\
POLR2A & -3.339 & 27.549 & 0.999 & 1.97 & $10 \mathrm{pg}-10 \mathrm{ng}$ \\
RPL13A & -3.067 & 27.381 & 0.998 & 2.15 & $1 \mathrm{pg}-100 \mathrm{ng}$ \\
PPIA & -3.327 & 28.560 & 0.999 & 2.00 & $1 \mathrm{pg}-100 \mathrm{ng}$ \\
HPRT1 & -3.234 & 28.451 & 0.999 & 2.05 & $10 \mathrm{pg}-10 \mathrm{ng}$ \\
\hline & & & & &
\end{tabular}

See Table II for gene name definitions.

and 24 included the majority of the candidate reference genes. Genes with lower expression levels had higher $\mathrm{Cq}$ values. Above 25 cycles, these genes were succinate dehydrogenase complex flavoprotein subunit A (SDHA), TATA box-binding protein (TBP), HPRT1 and RNA polymerase II subunit A (POLR2A). The remaining 14 candidate reference genes were highly expressed below 25 cycles. The expression levels of the 18 candidate reference genes did not depend on the sex, age, tumor stage or grade of the TCC samples. This result was consistent with the results of a previous study (17).

An ideal reference gene is required to meet the following criteria: i) Is usually abundant in studied tissues that can be reliably examined in all specimens; and ii) exhibits as little as possible expression variation across the tissue-specific sample set investigated. For the first criterion, genes with $\mathrm{Cq}$ values $>25$ cycles were arbitrarily selected for the exclusion of potential reference genes from subsequent evaluation. SDHA, TBP, HPRT1 and POLR2A exhibited lower expression levels in the TCC samples and were excluded following an evaluation of the reference genes (Fig. 2).

Stability of the expression of the candidate reference genes in TCC sample $\triangle C q$ analysis. The remaining 14 genes had different transcript level ranges over all the specimens investigated. The present study used matched cancerous and non-cancerous TTC samples, so the Cq of the normal sample minus the $\mathrm{Cq}$ of the corresponding cancer sample $(\Delta \mathrm{Cq})$ reflected the variation in the candidate reference genes between individuals (Fig. 3). For the second criterion, a $\Delta \mathrm{Cq}$ value $>1.9$ was arbitrarily selected to eliminate certain candidate genes 
Table IV. NormFinder analysis of 10 reference genes.

\begin{tabular}{clclclc}
\hline Rank order & Gene name & $\begin{array}{c}\text { Stability value } \\
\text { in all samples }\end{array}$ & Gene name & $\begin{array}{c}\text { Stability value } \\
\text { in normal samples }\end{array}$ & Gene name & $\begin{array}{c}\text { Stability value } \\
\text { in cancer samples }\end{array}$ \\
\hline 1 & UBC & 0.168 & RPS23 & 0.196 & RPS23 & 0.183 \\
2 & UBB & 0.185 & ATP5B & 0.203 & GAPDH & 0.233 \\
3 & TPT1 & 0.186 & TPT1 & 0.296 & ACTB & 0.249 \\
4 & RPS23 & 0.187 & GAPDH & 0.327 & RPS13 & 0.289 \\
5 & ATP5B & 0.194 & RPS13 & 0.339 & TPT1 & 0.290 \\
6 & RPL13A & 0.205 & RPL13A & 0.426 & ATP5B & 0.346 \\
7 & RPS13 & 0.207 & CFL1 & 0.592 & CFL1 & 0.374 \\
8 & GAPDH & 0.215 & ACTB & 0.602 & RPL13A & 0.467 \\
9 & ACTB & 0.230 & UBC & 0.628 & UBC & 0.493 \\
10 & CFL1 & 0.241 & UBB & 0.807 & UBB & 0.745 \\
\hline
\end{tabular}

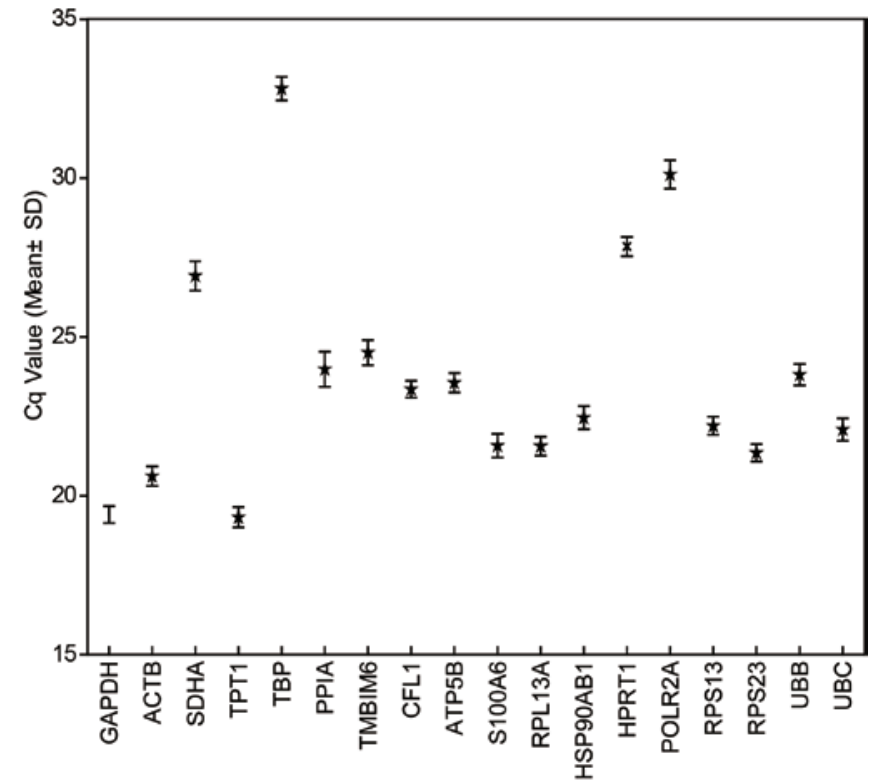

Figure 2. Expression levels of the 18 candidate reference genes in the transitional cell carcinoma samples. Cq, cycle threshold. SD, standard deviation. See Table II for gene name definitions.

from further consideration. In accordance with this analysis, GAPDH, peptidyl-prolyl isomerase A (PPIA), transmembrane BAX inhibitor motif-containing 6 (TMBIM6), heat-shock protein $90-\alpha$ family class B member 1 HSP90AB1 and S100 calcium-binding protein A6 (S100A6) were excluded from the subsequent calculations. Nevertheless, because GAPDH has been used in a number of studies, it was decided that GAPDH would be evaluated in the list of candidate reference genes.

A paired Student's t-test was also used to examine differences in the expression of candidate reference genes between the matched non-malignant and malignant specimens. There were significant differences in gene expression for all of the investigated reference genes, other than $\beta$-actin (ACTB) $(\mathrm{P}=0.0763), \mathrm{RPS} 23(\mathrm{P}=0.0746)$, TPT1 $(\mathrm{P}=0.064)$ and $\mathrm{RPS} 13$ $(\mathrm{P}=0.0532)$. The expression of cofilin 1 (CFL1), GAPDH, S100A6, HSP90AB1, TMBIM6 and PPIA were all significantly increased in the malignant samples $(\mathrm{P}<0.001)$ compared with the non-malignant groups. This result was similar to that obtained for the $\Delta \mathrm{Cq}$ values.

According to above analysis, 8 genes were excluded from the 18 candidate reference genes in the matched malignant and non-malignant sample pairs. The remaining 10 reference genes underwent further analysis with the mathematical software programs geNorm (11), NormFinder (12) and BestKeeper (13).

GeNorm analysis. In view of the precondition that the ratio of the expression level of suitable reference genes must be invariable under all experimental conditions, GeNorm calculates the M-value of a single gene by gradually ruling out the highest-scoring reference gene, then repeatedly recalculating in order to obtain the optimal value.

The stability of the expression for the 10 candidate genes is ordered according to the M-value, with the lowest M corresponding to the most stably expressed genes. $M=1.5$ is the threshold recommended for all selected reference genes. The genes with the smallest M-value were RPS13 and RPS23 (0.58 and 0.533 , respectively), which were the most stable genes in all 70 TCC and 35 non-malignant TCC samples in the present study. The order of gene stability, from the most to least stable gene was: RPS23, RPS13, TPT1, RPL13A, GAPDH, ATP synthase, $\mathrm{H}^{+}$transporting, mitochondrial F1 complex, $\beta$-polypeptide (ATP5B), CFL1, ACTB, UBC and UBB (Fig. 4A and C). In the malignant group, RPS23 and RPS13 were still the most stable genes, with UBB and UBC again exhibiting the largest M-value. For the rest of the selected reference genes, the order of expression stability, from the most to least stable, was: TPT1, GAPDH, ACTB, CFL1, ATB5B and RPL13A (Fig. 4E). The M-values of the ten genes were $<1$ in the TCC samples, below the default threshold of 1.5 , suggesting a relatively high stability of expression of the selected genes (Fig. 4).

GeNorm also offers a normalization factor for determining the optimal number of candidates by calculating the pairwise value of variation, V. For the selection of RT-qPCR reference genes, 0.2 is recommended as the threshold value. A total of 4 genes were required for good normalization in the all-TCC sample group, whereas two genes and four genes were 
Table V. BestKeeper analysis of ten candidate reference genes.

\begin{tabular}{llclclc}
\hline Rank order & Gene name & $\begin{array}{c}\text { R-value (P-value) } \\
\text { in all samples }\end{array}$ & Gene name & $\begin{array}{c}\text { R-value (P-value) } \\
\text { in normal samples }\end{array}$ & Gene name & $\begin{array}{c}\text { R-value (P-value) } \\
\text { in cancer samples }\end{array}$ \\
\hline 1 & RPS23 & $0.967(0.01)$ & ATP5B & $0.973(0.01)$ & TPT1 & $0.97(0.01)$ \\
2 & TPT1 & $0.964(0.01)$ & RPS23 & $0.971(0.01)$ & RPS23 & $0.963(0.01)$ \\
3 & ATP5B & $0.964(0.01)$ & TPT1 & $0.968(0.01)$ & ACTB & $0.955(0.01)$ \\
4 & UBC & $0.954(0.01)$ & UBC & $0.963(0.01)$ & GAPDH & $0.954(0.01)$ \\
5 & GAPDH & $0.95(0.01)$ & GAPDH & $0.96(0.01)$ & RPL13A & $0.948(0.01)$ \\
6 & RPS13 & $0.947(0.01)$ & RPS13 & $0.95(0.01)$ & RPS13 & $0.947(0.01)$ \\
7 & RPL13A & $0.942(0.01)$ & ACTB & $0.939(0.01)$ & ATP5B & $0.946(0.01)$ \\
8 & ACTB & $0.938(0.01)$ & RPL13A & $0.933(0.01)$ & UBC & $0.942(0.01)$ \\
9 & CFL1 & $0.921(0.01)$ & CFL1 & $0.926(0.01)$ & CFL1 & $0.921(0.01)$ \\
10 & UBB & $0.908(0.01)$ & UBB & $0.92(0.01)$ & UBB & $0.878(0.01)$ \\
\hline
\end{tabular}

The coefficient of correlation, $\mathrm{R}$, demonstrates gene expression variation, which is determined by calculating the coefficient of variance and standard deviation of the Cq set. See Table II for gene name definitions.

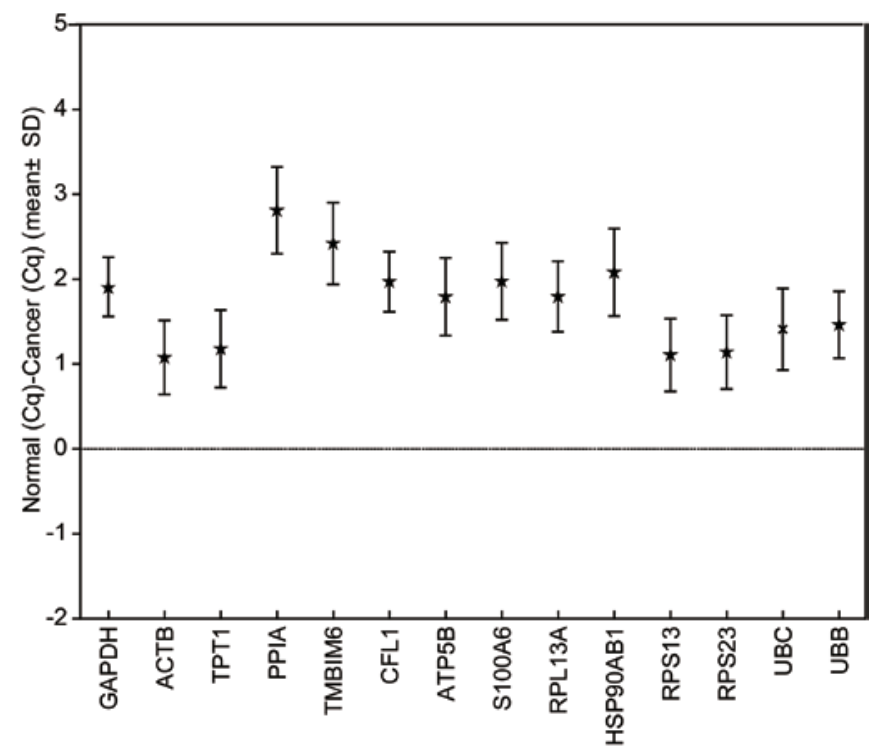

Figure 3. Gene expression level difference between matched non-malignant and malignant samples. The asterisks represent the mean $\Delta \mathrm{Cq}$ values $[\mathrm{Cq}$ (non-malignant)-Cq (malignant)]. Cq, cycle threshold. SD, standard deviation. See Table II for gene name definitions.

required in the non-malignant and malignant group, respectively (Fig. 4B, D and F).

NormFinder analysis. NormFinder is also a freely available tool for evaluating gene stability for normalization, ranking the candidate reference genes according to the output value, M. Those with the lowest M-value are considered to be the most stable reference genes. This approach combines the intergroup and intragroup expression variations of the candidate reference genes. The results calculated using NormFinder are listed in Table IV.

In this analysis, RPS23 was ranked the highest in the non-malignant and malignant groups. This was unexpected, as UBC was the most stably expressed candidate gene in all
Table VI. Final ranking of 10 candidate reference genes in all transitional cell carcinoma samples.

\begin{tabular}{clllll}
\hline Rank & \multirow{2}{*}{ Cq } & $\begin{array}{c}\text { Ge } \\
\text { Norm }\end{array}$ & $\begin{array}{c}\text { Norm } \\
\text { Finder }\end{array}$ & $\begin{array}{c}\text { Best } \\
\text { Keeper }\end{array}$ & Overall \\
\hline 1 & ACTB & RPS23 & UBC & RPS23 & RPS23 \\
2 & RPS13 & RPS13 & UBB & TPT1 & TPT1 \\
3 & RPS23 & TPT1 & TPT1 & ATP5B & RPS13 \\
4 & TPT1 & RPL13A & RPS23 & UBC & UBC \\
5 & UBC & GAPDH & ATP5B & GAPDH & ACTB \\
6 & UBB & ATP5B & RPL13A & RPS13 & ATP5B \\
7 & ATP5B & CFL1 & RPS13 & RPL13A & UBB \\
8 & RPL13A & ACTB & GAPDH & ACTB & RPL13A \\
9 & CFL1 & UBC & ACTB & CFL1 & GAPDH \\
10 & GAPDH & UBB & CFL1 & UBB & CFL1 \\
\hline
\end{tabular}

Cq, cycle threshold. See Table II for gene name definitions.

the TCC samples, which was the exact opposite of the result obtained using geNorm. This discrepancy may reflect differences in the algorithm utilized. Broadly speaking, the best two-gene combination from the NormFinder program was that of ATP5B and RPS23 (M=0.081). M values reflect gene expression stability.

BestKeeper analysis. BestKeeper is a commonly used software program that evaluates the stability of reference gene expression directly using raw $\mathrm{Cq}$ values. The BestKeeper index uses $\mathrm{R}$ to reveal gene expression variation, which is determined by calculating the coefficient of variance and standard deviation of the $\mathrm{Cq}$ set.

According to the BestKeeper analysis, $\mathrm{RPS} 23(\mathrm{R}=0.967)$, TPT1 $(R=0.964)$ and ATP5B $(R=0.964)$ demonstrated the optimal associations $(\mathrm{P}<0.001$ for the TCC specimens) (Table V). ATP5B, RPS23 and TPT1 were the optimal candidate 


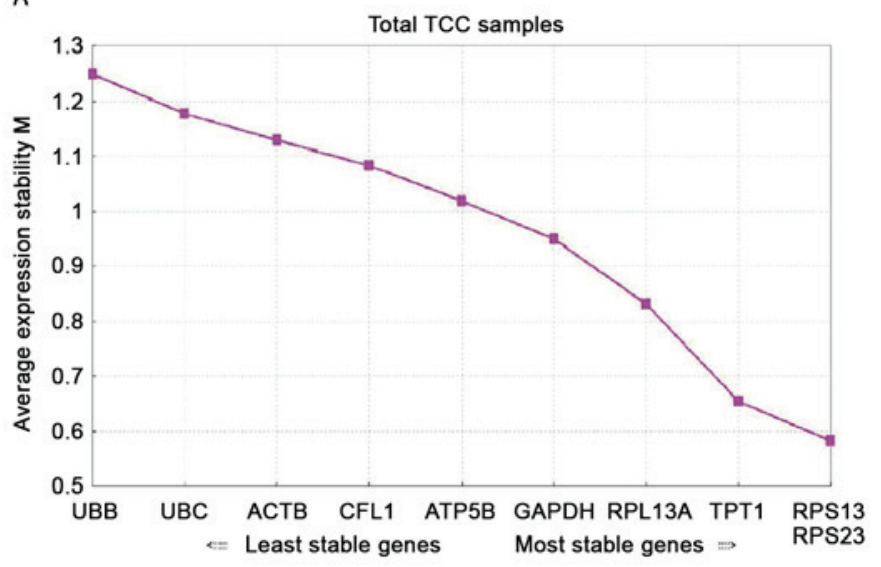

C

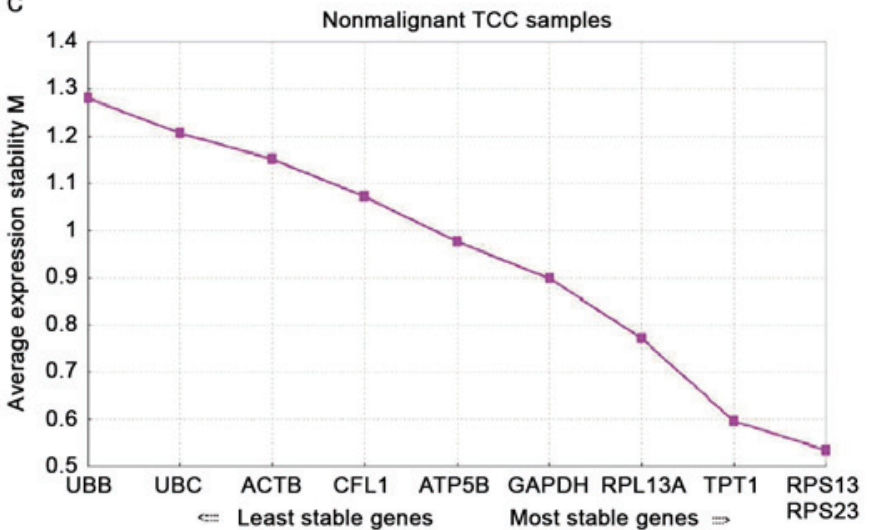

E

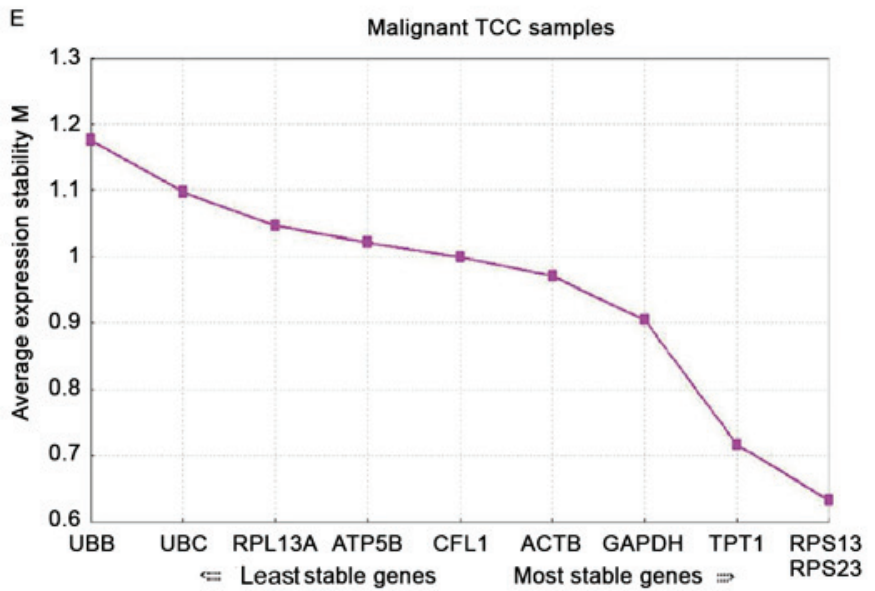

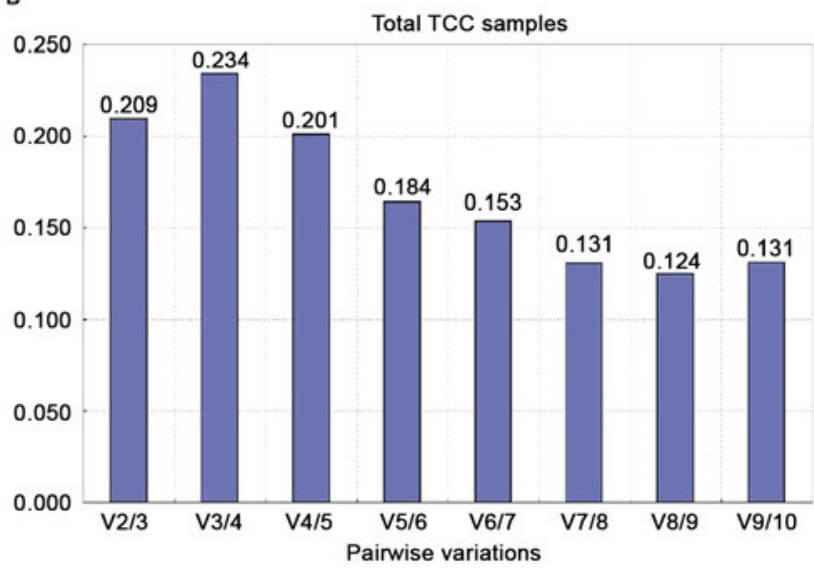

D

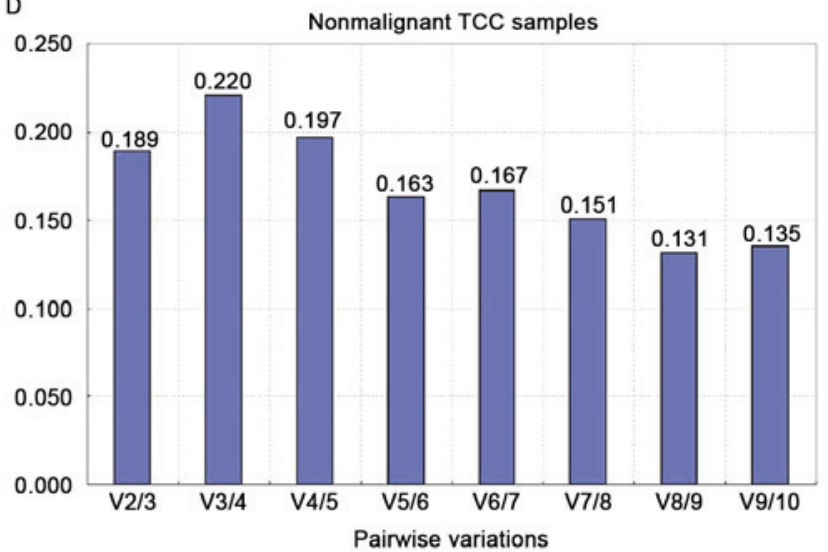

$\mathrm{F}$

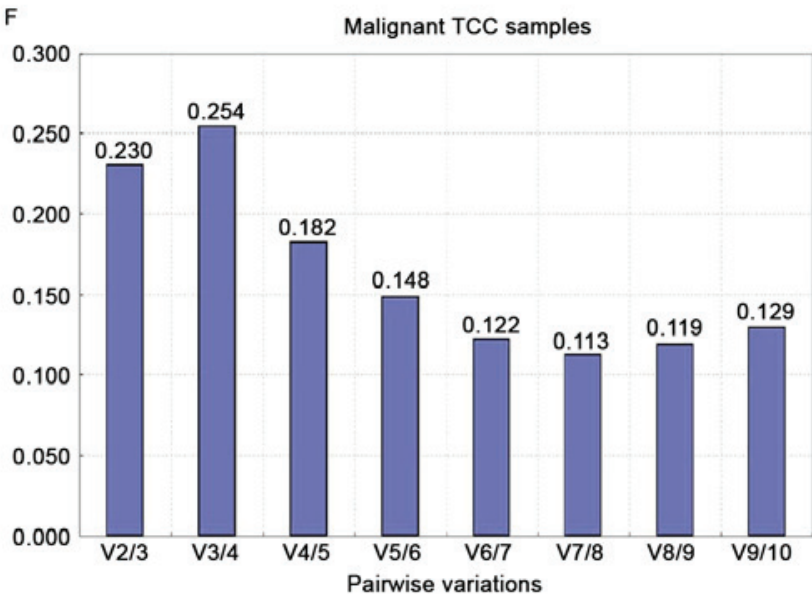

Figure 4. Ranking of gene stability and determination of the optimal number of reference genes evaluated using geNorm. The results are presented according to the protocol for the geNorm program output file. (A) The highest-scoring gene was gradually eliminated by calculating the average M value for all TCC samples. (B) Determination of the optimal number of reference genes for the normalization of all TCC samples. (C) The highest-scoring gene was gradually eliminated by calculating the average $\mathrm{M}$ value for non-malignant TCC samples. (D) Determination of the optimal number of reference genes for the normalization of non-malignant TCC samples. (E) The highest-scoring gene was gradually eliminated by calculating the average M value for malignant TCC samples. (F) Determination of the optimal number of reference genes for the normalization of malignant TCC samples. TCC, transitional cell carcinoma. See Table II for gene name definitions.

reference genes in the non-malignant group. TPT1, RPS23 and ACTB were the top three genes in the malignant group.

Final ranking of the selected candidate reference genes. Considering the discrepancies among the four algorithms, a method was used to calculate the final ranking of candidate reference genes (24). Specifically, the geometric mean for each gene was calculated using the four ranking numbers produced by $\Delta \mathrm{Cq}$ analysis (of the corresponding non-malignant and malignant samples) using geNorm, NormFinder and BestKeeper. The genes with the smallest geometric means were identified as the most stable (Table VI). 
RPS23 was identified as the most stable single gene from the analysis, with RPS23, TPT1 and RPS13 being the optimal reference gene set in all the TCC samples. RPS13, RPS23 and TPT1 were also suitable reference genes for the matched non-malignant and malignant samples. The overall rank for the matched non-malignant and malignant samples is not presented, as the final ranking for all of the TCC samples illustrates this point.

\section{Discussion}

The aim of the present study was to identify the most stable reference genes to ensure credible evaluation of the transcript levels of genes of interest in human bladder cancer, specifically TCC. A total of 18 candidate reference genes were selected from a variety of databases and previous publications that investigated reference gene expression profiles (11-16); these selected genes were assessed using SYBR-Green RT-qPCR in 35 pairs of matched non-malignant and malignant samples. The results of the present study demonstrate that the most stable reference gene was the rarely used RPS23, and the optimal three-gene combination, RPS23, TPT1 and RPS13, was found to be optimal for all the TCC samples, on the basis of the results of the four algorithms used.

To obtain reliable results in the RT-qPCR analysis, a concerted effort was made to ensure that each of the following criteria was met: i) All the bladder cancer samples were TCC, which is the most frequent subtype, and each sample included a malignant specimen and corresponding non-malignant specimen; ii) according to the MIQE guidelines (7), the quality and quantification of RNA and the specificity of each gene primer was strictly controlled; iii) a careful selection was carried out of 18 candidate genes from previous databases and publications reporting stable gene expression profiles (11-16); and iv) three commonly used software programs, geNorm, NormFinder and BestKeeper were combined with Student's t-test and $\Delta \mathrm{Cq}$ analysis to evaluate the candidate reference genes.

To the best of our knowledge, there are two published studies on the selection of the optimal reference gene for bladder cancer, with only one having been performed using SYBR-Green (12), although it was not assessed with matched sample pairs and different mathematical algorithms. Although the other study used matched sample pairs (14 pairs), it was performed with TaqMan methods (15). In general, the SYBR-Green method is cheaper and easier to use than TaqMan, but can lead to false-positive results owing to the presence of non-specific products, such as primer-dimers; these incorrect and shifted data may ultimately diminish the accuracy (24). In the present study, this potential problem was controlled for by the running of agarose gels and checking the $T_{m}$ values to guarantee the accuracy of the unique qPCR product.

A large number of factors influence the expression level of genes in tumor tissues; these include the type, age, stage and grade of the tumor samples investigated. In the present study, the results indicate that the expression of none of the candidate reference genes was dependent on the sex, tumor stage or grade of the TCC samples. An ideal reference gene is one that is usually abundant in the studied tissues, meaning that it can be reliably measured in all of the studied materials. Thus, the $\mathrm{Cq}$ value of a gene was arbitrarily selected at $>25$ cycles for the exclusion of potential internal genes. Accordingly, SDHA, TBP, HPRT1 and POLR2A were excluded following evaluation of the reference genes in this analysis.

To compare the evaluation results, the same candidate reference genes as those selected in two published studies $(12,15)$ were selected. In the study by Andersen et al (12), HSP90AB1, TMBIM6 and ATP5B were reported to be the optimal reference genes. However, TBP and SDHA were the optimal reference genes in the study by Ohl et al (15). These genes in these two studies are not included in the results of the present study, a discrepancy that may have arisen owing to differing qPCR conditions, mathematical methods and, most importantly, TCC samples. As mentioned in the study by Ohl et al (14), when using a greater number of matched pairs of non-cancerous and cancerous samples, the accuracy of the result was increased. Subsequently, the present study used a paired Student's t-test and $2^{-\Delta \Delta C q}$ analysis to examine significant differences between the expression levels of the candidate reference genes in the non-malignant and malignant sample pairs. Consequently, SDHA, TBP, HPRT1, POLR2A, CFL1, S100A6, HSP90AB1, TMBIM6 and PPIA were excluded from further analysis.

The readily available software programs geNorm, NormFinder and BestKeeper were used to evaluate the optimal genes from a set of candidate reference genes (23). Of these three programs, geNorm and NormFinder are able to provide the optimal combination of reference genes. In the case of NormFinder analysis, it is also possible to obtain the optimal single reference gene. NormFinder combines the intergroup and intragroup expression variations of the candidate reference genes, reducing the bias of the result. Unlike other algorithms, the output of BestKeeper has the capacity to analyze $<10$ selected reference genes. The results obtained using these programs differed somewhat, although this was generally acceptable as each program used different statistical algorithms. The more programs used, the more promising the results obtained in the evaluation of reference genes. Despite the slight discrepancies between the programs, the results from all of these algorithms indicate that RPS23 was the optimal single gene for normalization, and RPS23, TPT1 and RPS13 comprised the optimal combination of reference genes for evaluating TCC samples.

In conclusion, the results of the present study demonstrate that RPS23 was the most stably expressed reference gene, with the three most stable genes, RPS23, TPT1 and RPS13, comprising the most suitable geneset for all bladder samples. These reference genes may be used for gene normalization in studies of TCC gene expression, which are important for seeking novel molecular markers for bladder cancer in the future.

\section{References}

1. Torre LA, Bray F, Siegel RL, Ferlay J, Lortet-Tieulent J and Jemal A: Global cancer statistics, 2012. CA Cancer J Clin 65: 87-108, 2015

2. Siegel RL, Miller KD and Jemal A: Cancer Statistics, 2015. CA Cancer J Clin 65: 5-29, 2015.

3. Kaufman DS, Shipley WU and Feldman AS: Bladder cancer. Lancet 374: 239-249, 2009. 
4. Babjuk M, Burger M, Zigeuner R, Shariat SF, van Rhijn BW, Compérat E, Sylvester RJ, Kaasinen E, Böhle A, Palou Redorta J, et al: EAU guidelines on non-muscle-invasive urothelial carcinoma of the bladder: Update 2013. Eur Urol 64: 639-53, 2013.

5. Nseyo UO and Lamm DL: Immunotherapy of bladder cancer. Semin Surg Oncol 13: 342-9, 1997.

6. van Rhijn BW, van der Poel HG and van der Kwast TH: Urine markers for bladder cancer surveillance: A systematic review. Eur Urol 47: 36-48, 2005.

7. Bustin SA, Benes V, Garson JA, Hellemans J, Huggett J, Kubista M, Mueller R, Nolan T, Pfaffl MW, Shipley GL, et al: The MIQE guidelines: Minimum information for publication of quantitative real-time PCR experiments. Clin Chem 55: 611-622, 2009.

8. Li YL, Ye F, Hu Y, Lu WG and Xie X: Identification of suitable reference genes for gene expression studies of human serous ovarian cancer by real-time polymerase chain reaction. Anal Biochem 394: 110-116, 2009.

9. Warrington JA, Nair A, Mahadevappa M and Tsyganskaya M: Comparison of human adult and fetal expression and identification of 535 housekeeping/maintenance genes. Physiol Genomics 2: 143-147, 2000.

10. Thellin O, Zorzi W, Lakaye B, De Borman B, Coumans B, Hennen G, Grisar T, Igout A and Heinen E: Housekeeping genes as internal standards: Use and limits. J Biotechnol 75: 291-295, 1999.

11. Vandesompele J, De Preter K, Patty F, Poppe B, Van Roy N, De Paepe A and Speleman F: Accurate normalization of real-time quantitative RT-PCR data by geometric averaging of multiple internal control genes. Genome Biol 3: RESEARCH0034, 2002.

12. Andersen CL, Jensen JL and Ørntoft TF: Normalization of real-time quantitative reverse transcription-PCR data: A model-based variance estimation approach to identify genes suited for normalization, applied to bladder and colon cancer data sets. Cancer Res 64: 5245-5250, 2004.

13. Pfaffl MW, Tichopad A, Prgomet C and Neuvians TP: Determination of stable housekeeping genes, differentially regulated target genes and sample integrity: BestKeeper-Excel-based tool using pair-wise correlations. Biotechnol Lett 26: 509-515, 2004.

14. Leduc V, Legault V, Dea D and Poirier J: Normalization of gene expression using SYBR green qPCR: A case for paraoxonase 1 and 2 in Alzheimer's disease brains. J Neurosci Methods 200 $14-19,2011$
15. Ohl F, Jung M, Radonić A, Sachs M, Loening SA and Jung K: Identification and validation of suitable endogenous reference genes for gene expression studies of human bladder cancer. J Urol 175: 1915-1920, 2006.

16. Radonić A, Thulke S, Mackay IM, Landt O, Siegert W and Nitsche A: Guideline to reference gene selection for quantitative real-time PCR. Biochem Biophys Res Commun 313: 856-862, 2004.

17. Kent WJ, Sugnet CW, Furey TS, Roskin KM, Pringle TH, Zahler AM and Haussler D: The human genome browser at UCSC. Genome Res 12: 996-1006, 2002.

18. Jacob F, Guertler R, Naim S, Nixdorf S, Fedier A, Hacker NF and Heinzelmann-Schwarz V: Careful selection of reference genes is required forreliable performance of RT-qPCR in human normal andcancer cell lines. PLoS One 8: e59180, 2013.

19. Zhou ZJ, Zhang JF, Xia P, Wang JY, Chen S, Fang XQ and Fan SW: Selection of suitable reference genes for normalization of quantitative real-time polymerasechain reaction in human cartilage endplate of the lumbar spine. PLoS One 9: e88892, 2014.

20. Cicinnati VR, Shen Q, Sotiropoulos GC, Radtke A, Gerken G and Beckebaum S: Validation of putative reference genes for gene expression studies in human hepatocellular carcinoma using real-time quantitative RT-PCR. BMC Cancer 8: 350, 2008.

21. Freeman WM, Walker SJ and Vrana KE: Quantitative RT-PCR: Pitfalls and potential. Biotechniques 26: 112-122, 124-125, 1999.

22. Pombo-Suarez M, Calaza M, Gomez-Reino JJ and Gonzalez A: Reference genes for normalization of gene expression studies in human osteoarthritic articular cartilage. BMC Mol Biol 9: 17, 2008.

23. Jacob F, Guertler R, Naim S, Nixdorf S, Fedier A, Hacker NF and Heinzelmann-Schwarz V: Careful selection of reference genes is required for reliable performance of RT-qPCR in human normal and cancer cell lines. PLoS One 8: e59180, 2013.

24. Chen D, Pan X, Xiao P, Farwell MA and Zhang B: Evaluation and identification of reliable reference genes for pharmacogenomics, toxicogenomics, and small RNA expression analysis. J Cell Physiol 226: 2469-2477, 2011.

25. Sobin LH, Gospodariwicz M and Wittekind C (eds): TNM classification of malignant tumors. U1CC 1nternational Union Against Cancer. 7th edition. Wiley-Blackwell, pp262-265, 2009. 